\title{
Do morphophonological exchange rules exist? A reply to DiCanio et al. (2020)
}

\author{
Paul de Lacy
}

\author{
Rutgers University - delacy@rutgers.edu
}

\begin{abstract}
DiCanio et al. (2020) (this volume) argue that San Martín Itunyoso Triqui has a morphophonological exchange (also called 'polarity'), where morphemes are realized by switching feature values: e.g. the bare root [anĩh] 'get dirty' is realized as [anĩ:] in the 1s, while the root [ani:] 'stop' is 1s [anĩh]. In this Reply, I seek to clarify how the descriptive use of 'exchange' relates to and differs from its meaning in phonological theories. I also show that the issue of whether exchanges exist is highly theory-dependent. For SPE, Lexical Phonology and Morphology, and single-level parallelist OT with opacity mechanisms, the IT forms do not provide evidence for exchange mechanisms. In contrast, a version of OT that lacks opacity mechanisms probably cannot generate the IT forms without an exchange mechanism. Issues facing the analyst, such as how to prove that exchanges exist, and which apparent exchanges one should expect to observe, are also discussed.
\end{abstract}

Keywords: morphophonological polarity; exchange rules; anti-faithfulness; opacity; Itunyoso Triqui

\section{Introduction}

DiCanio, B. Martínez Cruz, Cruz Martínez, and W. Martínez Cruz (2020) (hereafter D) newly report the alternations in (1), from San Martín Itunyoso Triqui (hereafter IT). Where there is a final long vowel in the bare root form, the '3rd person proximate singular' (3TS) morpheme is realized as an $\left[\mathrm{h}^{3}\right](1 \mathrm{a}, \mathrm{b})$; however, where there is a [ $\mathrm{h}]$ in the bare root, the $3 \mathrm{TS}$ form appears with a long vowel $(1 \mathrm{c}, \mathrm{d})$. Generalizing, the $3 \mathrm{TS}$ morpheme seems to be realized by exchanging vowel length and [ $\mathrm{h}]$.

(1) Itunyoso Triqui 'glottal toggling' (D:Table 17)
a. $\left[\mathrm{ri}^{32}\right]$ 'to take out'
$\left[\mathrm{rih}^{3}\right]\{3 \mathrm{TS}\}$
b. $\left[\mathrm{a}^{3} \mathrm{ja}^{32}\right]$ 'to read'
$\left[\mathrm{a}^{3} \mathrm{jah}^{3}\right]\{3 \mathrm{TS}\}$
c. $\left[\mathrm{a}^{3}\right.$ tof $\left.^{3}\right]$ 'to laugh'
$\left[\mathrm{a}^{3}\right.$ to: $\left.:^{3}\right]\{3 \mathrm{TS}\}$
d. $\left[\mathrm{rã} \mathrm{h}^{4}\right]$ 'to buy'
$\left[\right.$ rã $\left.^{3}\right]\{3 \mathrm{TS}\}$

The 3TS morpheme is not unique in IT; similar alternations are seen with the 1st person singular suffix (1S) and a nominalizer. Importantly, D takes pains to show that the alternations seen in (1) are well attested and productive.

Similar morphophonological 'exchanges' have been identified and discussed by Anderson \& Browne (1973) and many others (see references in D and de Lacy 2012). However, de Lacy (2012), among others, argues that there is no compelling evidence for morphophonological exchange mechanisms (also see Bye 2006, Trommer 2007, Wunderlich 2012). So, does IT provide such evidence?

This Reply argues that the answer to this question depends on what the question means. 'Exchange' has both descriptive/taxonomic and theoretical meanings, and the theoretical meanings are theory-specific. In its descriptive sense, the IT alternations can be called 'exchanges' (see section 2). However, the 
theoretical issue is, for any specific theory, whether that theory can generate the observations about IT reported in D without employing 'exchange mechanisms'. What exactly 'exchange mechanisms' are depends on the theory. In the SPE framework (Chomsky \& Halle 1968), Anderson \& Browne (1973) propose an exchange mechanism that consists of rules of the form $[\alpha \mathrm{F}] \rightarrow[-\alpha \mathrm{F}]$ with a specific morphological environment ('exchange rules'). In Optimality Theory (Prince \& Smolensky 2004), Alderete (2001) proposes trans-derivational anti-faithfulness constraints as an exchange mechanism; de Lacy (2012) identifies additional constraint types. So, focusing on Anderson \& Browne (1973)'s proposal, the present question must be recast as "Must every SPE rule system that generates the IT alternations include an exchange rule?" In contrast, for Alderete (2001)'s proposal in single level parallelist OT (Prince \& Smolensky 2004), the question is "Must every ranking that generates the IT alternations include a transderivational anti-faithfulness constraint?"

For the theoretical questions, I argue that the IT alternations do not provide evidence for exchange rules in SPE (section 3), or anti-faithfulness constraints in single-level parallelist OT with opacity mechanisms (section 4). However, there are versions of such theories which do require such exchange mechanisms.

This Reply then turns to more general questions for the analyst: how do we prove that exchange mechanisms are necessary (section 5)? If it is possible to generate apparent exchanges without exchange mechanisms, should we expect to see any type of apparent exchange (section 6)? I conclude with comments on the analyst's broader task in regard to morphophonological exchanges (section 7).

\section{What is an exchange?}

The term 'morphophonological exchange' is ambiguous: like many terms in linguistics, it has both taxonomic (descriptive) and computational meanings.

In the description/taxonomy of languages, 'morphophonological exchange' can be used to describe two pairs of forms, where each pair includes forms that differ in terms of a single morpheme $\mathrm{M}$. One pair consists of a form that lacks $M$ and contains $[\alpha]$ (a feature, segment, prosodic element, or substructure) and another form that contains $\mathrm{M}$ and has a corresponding $[\beta]$; the other pair has a form with $[\beta]$ and an $\mathrm{M}$ form with a corresponding $[\alpha]$. In IT, for example, there are two pairs: [anĩ:] 'stop' $\sim$ [anĩ $]\{1 s\}$ and [anĩh] 'get dirty' [anĩ:] \{1s\} (D:Table 10). The former pair has [:] in the bare root, but a corresponding [6] in the $1 \mathrm{~s}$, while the latter pair has the opposite. So, D's IT observations are very reasonably described as an exchange.

For Generative transformational theories, there is no straightforward analogue to the descriptive/taxonomic definition of 'exchange'. D focus attention on two theories - SPE (Chomsky \& Halle 1968) and single-level parallelist OT (Prince \& Smolensky 2004) - so I will do the same here (see section 4 for discussion of other theories).

In SPE, there is no formally definable direct connection between the phonological outputs [anĩ:] 'stop' and [anĩh] 'stop $+1 \mathrm{~s}$ '. Instead, the theory expresses their connection indirectly - both outputs include a common underlying form, and both undergo transformations via the same rule system. In section 3, I will argue that the underlying form is /anĩ/, so /anĩ/ $\rightarrow$ anĩ:] and /anĩ $+h / \rightarrow$ [anĩ $]$.

Consequently, any theoretically meaningful definition of 'exchange' in SPE (and other similar theories) requires that definition to be cast in terms of derivation. As discussed by Anderson \& Browne (1973), in SPE a morphophonological exchange can be defined as a morphologically conditioned point in the derivation where both the mappings $[+\mathrm{F}] \rightarrow[-\mathrm{F}]$ and $[-\mathrm{F}] \rightarrow[+\mathrm{F}]$ occur (where $\mathrm{F}$ is a feature). 'Point in the derivation' refers to the output of a rule that effects such a mapping. Anderson \& Browne (1973) and Gregerson (1974) identify rules that can implement feature exchange as having the form $\left.[\alpha \mathrm{F}] \rightarrow[-\alpha \mathrm{F}] / \gamma \_\delta\right]_{\mathrm{M}}$, where a feature value is changed into its opposite in a specific morphological context $(\mathrm{M})$, potentially conditioned by its phonological environment $(\gamma, \delta)$. For example, Gregerson (1974:106) proposes a rule (slightly adapted here) [ $\alpha$ voice $] \rightarrow[-\alpha$ voice $] /$ +PLURAL to generate an apparent exchange in the DhoLuo plural (for discussion of this case, see de Lacy 2012). 
So, the question of whether IT has a morphophonological exchange is, in the SPE framework, adopting Anderson \& Browne (1973)'s proposal, a question of whether an exchange rule is required to generate the observed forms.

In single-level parallelist OT, 'morphophonological exchange' requires a different definition because OT does not have rules. It also makes no sense to say "at some point in the derivation $[+\mathrm{F}] \rightarrow[-\mathrm{F}]$ and $[-\mathrm{F}] \rightarrow[+\mathrm{F}]$ " because there is no multi-step derivation: there are only the input and output candidates and correspondence relations (McCarthy \& Prince 1995). Instead, one might define an exchange as involving a mapping to a winner in which $/+\mathrm{F} / \rightarrow[-\mathrm{F}]$ and $/-\mathrm{F} / \rightarrow[+\mathrm{F}]$ in a particular morphological environment. The mapping could be from any form to which faithfulness constraints can refer - e.g. an input, derivational base, paradigmatic base, or opaque form (see Tesar 2013 for relevant discussion). de Lacy (2012) argues that such mappings can be effected if there are particular classes of constraints, notably transderivational anti-faithfulness constraints (Alderete 2001), but also others like MORPHDISF.

So, the question of whether IT has a morphophonological exchange is, in OT, adopting Alderete (2001)'s proposal, a question of whether transderivational anti-faithfulness constraints are required to generate the observed forms.

I have taken pains to distinguish the descriptive/taxonomic and theoretical definitions of 'exchange' because it is enticing to infer from a descriptive exchange that for any theory there must be mappings of the type $/ \alpha / \rightarrow[\beta]$ and $/ \beta / \rightarrow[\alpha]$. Instead, what exactly a descriptive exchange requires of any particular theory must be evaluated in terms of that theory: i.e. what are the computational mechanisms that the theory requires in order to generate the observed output forms and their inferred derivations?

\section{Are there exchange rules in IT?}

D discuss two theories: SPE and OT. So, I will start with the SPE question: does IT requires an exchange rule?

'Requiring an exchange rule' means that every rule system that could generate the IT forms includes an exchange rule, and there is no rule system that lacks exchange rules and generates the IT forms. There are therefore two tasks for the analyst. One is to demonstrate that there is some rule system with an exchange rule that generates the observed forms. The other is to demonstrate that every such rule system includes an exchange rule - a much more complex task.

In contrast, demonstrating that IT does not provide evidence for exchange rules, only requires showing that there is some rule system that lacks exchange rules yet generates the IT forms. This is the task undertaken here.

It is important to note that I leave tone out of the analyses and transcriptions below. Even though tone is contrastive and there are complex tonal processes in IT, the exchange part of the problem is segmental, so tone will not be discussed until section 3.5.

It is also important to note that a good deal of the analysis here is inspired by proposals and suggestions in D (especially §3.2.1). I will note similarities to and differences from D's proposals in the discussion below; there is a more detailed discussion of D's rule-based analysis in section 5.

The IT word-final syllable, which is stressed, is always bimoraic CV: or CVC (D, §2.2.1). So, what happens to underlying short vowels that emerge in final position? While many resolutions are possible (Bye \& de Lacy 2008), I will assume here, in agreement with D (\$2.2), that underlying final short vowels lengthen: i.e. $/ \ldots \mathrm{CV} / \rightarrow[\ldots \mathrm{CV}:]^{1}$

\footnotetext{
${ }^{1}$ I follow D in assuming that vowel lengthening does not occur when consonantal enclitics are attached: e.g. /anĩ $+\mathrm{h} / \rightarrow$ anĩh], *[anĩ:h]. However, DiCanio (2008:24) states that "when personal enclitics attach, they do not prevent final vowel lengthening on the preceding root vowel"; if this is the case, the analysis would need to be revised.
} 
In SPE such rule-derived regularity allows lexical entries to be simplified so that no lexical entry has a final /V:/ - all underlying forms end in a short vowel. So, [anĩ:] 'stop' has the underlying form /anĩ/, and not /anĩ:/, agreeing with D (Table 24).

On inspecting the inflected form [anĩ $]$ 'stop+1s', the only part of the surface form that does not belong to the underlying form of the root is [h]. It therefore seems reasonable to assume that the learner will conclude that the underlying form of the $1 \mathrm{~s}$ is $/ \mathrm{h} /$. The same conclusion can be made for the 3TS enclitic (D, $\S 2.5)$.

Some input $\rightarrow$ output transformations that are of central interest in the IT exchange are given in (2).

(2) SPE Analysis: Input $\rightarrow$ Output
a. $/ \mathrm{V} / \rightarrow[\mathrm{V}:]$
/anĩ// $\rightarrow$ [anĩ:] 'stop'
b. $/ \mathrm{V}+\mathrm{h} / \rightarrow[\mathrm{Vh}]$
/anĩ $+\mathrm{h} / \rightarrow$ [anĩh] 'stop+1s'
c. $/ \mathrm{Vh}+\mathrm{h} / \rightarrow[\mathrm{V}:]$
/anĩh $+\mathrm{h} / \rightarrow$ [anĩ:] 'get dirty+1s'

The challenge is now clear: how does $/ \mathrm{Vh}+\mathrm{h} /$ surface as $[\mathrm{V}:]$ ? A clue is provided by roots that surface with final glottal stops.

\subsection{Class I glottal-final roots}

There are two classes of roots that end in glottal stops on the surface. For class I roots, the encliticization of /h/ produces a vowel between the glottal stop and [ $\mathrm{h}]$, as in (3).

Type I glottal-final roots (from D:Table 11(b))
a. [kĩ?] 'to stink'
[kĩ?ĩh] 'to stink+1s'
b. [nano?] 'to look for'
[nano?oh] 'to look for $+1 \mathrm{~s}$ '
c. [narã?] 'to pick up (mass N.)'
[narãrã $]$ 'to pick up+1s'

I will assume that the additional vowel is an epenthetic copy vowel (Hall 2011). So, the transformation involves an epenthesis process: e.g. $/ \mathrm{kĩ} \mathrm{P}+\mathrm{h} / \rightarrow[\mathrm{kĩ} \mathrm{rinh}]$. The motivation for epenthesis is clear: IT only allows one consonant per coda, so the form at concatenation [kĩ?h] must be altered. ${ }^{2}$

\section{$3.2 / \mathrm{h} /$-final stems}

It is now possible to ask about underlying /h/-final stems, such as /anĩh/ 'get dirty'. Such stems face the same problem as $/ \mathrm{R} /-$ final stems: concatenation of $/+\mathrm{h} /$ will produce a complex coda. The epenthesis rule mentioned above will apply, so just as $/ \mathrm{kĩ}+\mathrm{h} / \rightarrow[\mathrm{kĩ} \Upsilon \underline{1} \hat{h}]$, /anĩh $+\mathrm{h} /$ should transform into [a.nĩ.hĩ $\mathrm{h}]$.

However, [a.nĩ.fĩ h] presents a problem that is not encountered with / $/$ /-final stems: unlike [?], [ $\mathrm{h}]$ is banned in onsets. This prohibition motivates a series of repairs. First, the onset [€] deletes: [a.nĩ. î $]^{3}{ }^{3}$ However, adjacent identical vowels are not permitted (DiCanio 2008:45-46), so they merge into a single long vowel: [a.nĩ:h]. However, final superheavy CV:C syllables are not permitted, so the final [h] deletes: [a.nĩ:]. The rule system is summarized in (4), showing derivations of examples of each stem type mentioned so far.

\footnotetext{
${ }^{2} \mathrm{D}$ refer to epenthesis as 'reduplication', and conclude that the forms as in (3) "cannot be correctly derived" in the serialist analysis they entertain ( $\mathrm{D}, \S 3.2 .1)$. As reduplication is the realization of a morpheme, and the copy vowels do not signal any additional semantic or syntactic information, I instead analyze them as generated by the phonological system: i.e. they are epenthetic segments, not the realization of reduplicants.

${ }^{3} \mathrm{D}(\S 3.2 .1)$ also entertain a deletion rule called "Root-final C deletion". The present rule differs in only referring to [6], and only applying in onsets. See section 5 for further discussion of D's deletion rule.
} 


\begin{tabular}{|c|c|c|c|c|c|c|}
\hline \multicolumn{7}{|c|}{ SPE Analysis: IT derivations } \\
\hline Underlying Form & /anĩ/ & /anĩ $+\mathrm{h} /$ & /anĩh/ & /anĩh+h/ & $/ \mathrm{k} \tilde{1} \mathrm{P} /$ & $/ \mathrm{k} \tilde{\mathrm{p}}+\mathrm{h} /$ \\
\hline Concatenation & anĩ & anĩก & anĩh & anĩhh & kĩ? & kĩ?h \\
\hline V\# lengthening & añ̃: & & & & & \\
\hline Epenthesis & & & & anĩhĩh & & kĩนîh \\
\hline Onset fi deletion & & & & anĩĩh & & \\
\hline VV merger & & & & anĩ:h & & \\
\hline Superheavy C deletion & & & & anĩ: & & \\
\hline "Surface Form & añ̃: & בanĩh & anĩh & anĩ: & kĩ? & kĩ̃îh \\
\hline
\end{tabular}

Whether there is need for extrinsic ordering in this rule system depends on the precise formulation of superheavy consonant deletion (SCD). With a general formulation like $\mathrm{C} \rightarrow \varnothing / \mathrm{VX} \#$, epenthesis must precede SCD otherwise $/ \operatorname{anĩh}+h / \rightarrow *[a n i \tilde{h}]$. However, if SCD is limited to $\mathrm{V}: \mathrm{C}$ clusters (i.e. $\mathrm{C} \rightarrow \varnothing / \mathrm{V}$ : $\#$ ), there is no need for extrinsic ordering. So, depending on the analytical details, it is possible that all rules apply just when their structural descriptions are met.

Formulations of the rules mentioned above are given in (5), abiding by SPE's original formalism. Later versions of SPE include formatives for syllable boundaries and metrical structure; expressing the rules with these formatives is not germane to the point of this Reply as such reformulations still do not necessitate exchange rules. ${ }^{4}$

$\begin{array}{ll}\text { SPE Analysis: Rules } & \\ \text { V\# lengthening: } & \mathrm{V} \rightarrow[+ \text { long]/\# } \\ \text { Epenthesis: } & \varnothing \rightarrow \mathrm{V} / \mathrm{C} \_\mathrm{C} \# \\ \text { Onset } \mathrm{h} \text { deletion: } & \mathrm{h} \rightarrow \varnothing / \mathrm{V}_{-} \mathrm{V} \# \\ \text { VV merger: } & \mathrm{V}_{\mathrm{i}} \rightarrow[+\mathrm{long}] / \mathrm{V}_{\mathrm{i}} ; \mathrm{V}_{\mathrm{i}} \rightarrow \varnothing / \mathrm{V}_{\mathrm{i}} \\ \text { Superheavy C deletion: } & \mathrm{C} \rightarrow \varnothing / \mathrm{V}_{-} \#\end{array}$

Crucially, the rules in (5) do not include an exchange rule. Even if future evidence shows that this rule system is inadequate for IT, there is a crucial analytical result here: rule systems can generate apparent exchanges without having an exchange rule.

Megan Crowhurst (p.c.) makes an observation about why IT appears to involve an exchange. IT's wordfinal nuclei are extremely limited: [V:] and [Vh] (ignoring [V?] for a moment). Since the phonological system has only two options to choose from, and has strong restrictions on how it resolves illicit structures, the result of any non-trivial concatenation will be an apparent exchange: $/ \mathrm{V}: /$ can map to nothing else but [Vh], while /Vh/ has nowhere to go except for [V:]. So, under the right conditions, extreme limitations on surface outcomes can mean that non-exchange processes naturally converge on similar outcomes to exchange mechanisms (see section 5 for further discussion). In this case, it is very fortunate that IT has a third permissible word-final nucleus: [V?]. While [V:] and [Vh] appear to exchange, /V?/ gives crucial insight into what the system is actually doing - i.e. employing distinct epenthesis and deletion processes.

More generally for the analyst, the rule system above identifies an important pitfall. If one observes that underlying $/ \alpha /$ becomes surface $[\beta]$ and underlying $/ \beta /$ becomes surface $[\alpha]$, this observation is not enough in an SPE framework to conclude that there is an exchange rule. The SPE exchange mechanism applies to a point in the derivation, so it must be demonstrated that there is a featural exchange takes place at the same stage of the derivations of both $/ \alpha / \rightarrow[\beta]$ and $/ \beta / \rightarrow[\alpha]$. The rule system above shows that an exchange can appear to occur without there being any such stage.

\footnotetext{
${ }^{4}$ For example, D (\$3.2.1) proposes that V-final lengthening is conditioned by stress (which is word-final), and propose a rule that adds a mora to the final syllable. Hewing to original SPE formalism, which lacks moras and syllables, the rule is conditioned here by a final word boundary. However, following D more closely, the rule could instead be expressed as $\mathrm{V}^{[+ \text {stress }]} \rightarrow[+$ long $] / \#$, though this rule would then be simplified to the one in (5) as stress predictably falls word-finally.
} 
At this point, it is important to pause and ask whether the rule system above is really adequate for IT. While it handles the basic alternations, does it fit within a full account of the larger IT phonological system?

On the positive side, it is interesting to note that the proposed rules' motivations can be determined from phonotactic generalizations. Vowel lengthening in /anĩ// $\rightarrow$ [a.nĩ:] is motivated by a requirement that stressed syllables be bimoraic, which is evident from phonotactics: all output forms have a word-final stressed heavy syllable. Onset [6] deletion is motivated by a ban on onset [ 6 ] - again evident from the distribution of [G] in surface forms. Echo epenthesis is motivated by a ban on complex codas, which are prohibited in output forms. Superheavy $\mathrm{C}$ deletion is motivated by a ban on superheavy V:C syllables, again observable in non-alternating wordforms. So, the learner does not have to learn much to end up with the rule system above; after establishing phonotactic generalizations, the learner only has to learn the responses to violations of those generalizations, none of which are remarkable from a typological standpoint. From an analytical point of view, there is no rule here whose sole purpose is to shoe-horn the analysis into working - the motivations for the rules are all independently observable, and are formally part of the grammar as Morpheme Structure Constraints.

On the other hand, it is important to be cautious about assuming too much certainty. While D and DiCanio (2008) provide a great deal of information about IT's phonology and phonetics, there is still not the depth of understanding and resources that would allow confident assertions about whether any rule system is descriptively adequate in regard to IT's entire phonological system. For example, it is possible that there are alternations that show that an epenthesis rule is not right, or that underlying vowel-final roots augment with a coda consonant, rather than lengthen. Ideally, there would be data showing mappings of every affix and enclitic in every relevant phonological and morphological environment. It is worth pointing out that de Lacy (2012) only made headway in analyzing the well-known DhoLuo plural exchange because there were five grammars of the language to draw on - a great deal more information than is available for IT. The rule system for IT above is therefore necessarily tentative - a point I will return to in section 7 .

Even so, D have identified two other important aspects of IT's phonological system that any analysis must address: how another type of glottal-final root behaves, and how certain morphemes fail to produce exchanges.

\subsection{Class II glottal-final roots}

IT has an additional class of roots that surface in bare form with a final [?]. When $/+h /$ is concatenated with these roots, epenthesis does not occur: instead, the / $\mathrm{h} /$ replaces the [?]. For example, [toko?] 'hang (tr)' has a 1s form [tokof] (D, Table 11). So, why does [nano?] have a 1s form [nano?of] while [toko?]'s 1s is [tokoh]?

There are a variety of analytical options, such as evoking lexical classes. Here I explore the idea that the difference is due to a distinction in underlying forms: i.e. the [?]s in [toko?] and [nano?] are underlyingly distinct.

For [toko?], the surface [?] is derived from a defective underlying segment, such as an empty root node /•/ (for examples relevant to exchanges, see de Lacy 2012 and references cited therein). Crucially, the defective segment is unable to motivate epenthesis; so /toko $+\mathrm{h} /$ maps to [tokof], where the [h] merges with the empty root node. There are two analytical options in SPE: one is to order a $/ \bullet \mathrm{h} / \mathrm{merger}$ rule before epenthesis (as below); the other is to formulate the epenthesis rule so that $/ \bullet /$ does not meet its structural description. If $/ \bullet /$ survives to the end of the derivation, it receives the features of a glottal stop. 


\begin{tabular}{|c|c|c|c|c|}
\hline Underlying Form & /toko•/ & /toko•-h/ & /nano?/ & /nano?-h/ \\
\hline Concatenation & toko• & toko•h & nano? & nano?h \\
\hline$\bullet \rightarrow \varnothing / \mathrm{C}$ & & tokoh & & \\
\hline Epenthesis & & & & nano?oh \\
\hline Default feature fill-in & toko? & & & \\
\hline Surface Form & toko? & tokoh & nano? & nano?oh \\
\hline
\end{tabular}

The analysis predicts consistency in how the roots react to both the 1S and 3TS: whether a root undergoes epenthesis or merger with the $1 \mathrm{~s}$, it will do the same with the $3 \mathrm{TS}$. D, $\S 2.5$ assert that this is true for many roots: e.g. [kĩ?] 'stink' undergoes epenthesis in the 1s [kĩ̃îh] and 3TS [kĩ̃îf].

\subsection{Morphemes without exchanges}

As discussed above, the rules' motivations can be found in generalizations about phonotactic distributions. However, evidence for the rules' resolutions - e.g. that avoidance of clusters induces epenthesis, or superheavy syllables are resolved by coda deletion - require alternations. Is there independent evidence for such resolutions in alternations involving other IT morphemes?

$\mathrm{D}, \S 2.3$ mentions the causative $/ \mathrm{t}(\mathrm{V})-/$, iterative $/ \mathrm{n}(\mathrm{V})-/$, and aspectual $/ \mathrm{k}(\mathrm{V})-/$. However, no examples are provided that would provide evidence for or against any of the rules above. IT does not have any suffixes (DiCanio 2008:24). However, there are a number of enclitics.

Two enclitics are segmentally /-h/ - the 1s and 3TS - which both undergo alternations described above. Unfortunately, some of the remaining enclitics do not provide any opportunity for alternations since their concatenation does not create any ungrammatical structures. For example, the 2 nd person singular enclitic has the form CVC, and so does not provide any opportunities for epenthesis or superheavy deletion: e.g. /ni?jah-re?/ $\rightarrow$ [ni?jahre?] 'drink-2SG.FAM' (DiCanio 2008:154). The same is true for the 3SG.MASC enclitic /-sih/ (DiCanio 2008:18; D:Table 5).

However, the first person dual suffix (1DU) appears to be a /?/ or $/ \bullet /-$ e.g. [kato:] 'shirt', [kato?] 'shirt+1DU' (D, Table 7). Unfortunately, the forms provided in D's Table 7 for the 1DU are not particularly probative. The 1DU attaches to /V/-final stems straightforwardly: e.g. /stĩ+?/ $\rightarrow$ [stĩ?] 'fingernail+1DU'. There is one example with final /h/: [snã?ãn] 'language' has the 1DU form [si-snũ?ũ?]. However, it is not clear here whether [si-snũ?ũ?] is suppletive (suggested by the replacement of the root's [ã]s with [ũ]s).

$\mathrm{D}, \S 3.2 .3$ mentions that emphatic marking on nouns involves suffixing a glottal stop. Interestingly, "no segmental toggling takes place - the glottal stop here replaces the final mora of the final syllable." Data from roots with final $/ \mathrm{h} /, / \mathrm{R} /$, and $/ \bullet /$ could potentially provide insight into the underlying form of the emphatic suffix/enclitic.

I have not identified evidence in DiCanio $(2008,2010)$ or D that shows what happens to subminimal $/ \mathrm{CV} /$ roots. Such alternations would confirm whether the prohibition on final short vowels is really resolved via lengthening, or some other method.

So, IT apparently has morphemes that do not evoke exchanges and may provide crucial insight into the adequacy of any analysis. However, with the current published sources, there is not enough information to make any analytical assertion with confidence at this time.

\footnotetext{
${ }^{5} \mathrm{D}, \S 2.4$ observes that of 105 glottal-final stems observed, 22 stems had epenthesis while 83 had merger. Furthermore, the stems with epenthesis could optionally be produced with merger instead. D further suggests that a process of intervocalic glottal stop lenition (or deletion) might be responsible for merger cases. Of course, while these observations suggest that phonology or lexicon is changing, they do not have any impact on the analytical issue of how to account for the different behavior of [toko?] and [nano?].
} 


\subsection{Tone}

Tone plays a major role in IT, and the enclitics that show apparent exchanges trigger significant tonal processes, as discussed by D. However, the morphemes do not display any tonal exchanges. The one potentially relevant issue is that tone conditions a segmental alternation for the 3TS morpheme.

3 TS stems that end in $/ \ldots \mathrm{V}^{3} /$ surface as $\left[\ldots \mathrm{V}::^{3}\right]$, not $\left[\mathrm{Vh}^{3}\right]:$ e.g. $/ \beta \tilde{1}^{3}+\mathrm{h}^{3} / \rightarrow\left[\beta \tilde{\mathrm{r}}:{ }^{3}\right], *\left[\beta \tilde{1} \mathrm{~h}^{3}\right]$ (D:Table 21). $/ \mathrm{V} /$-final forms with other tones surface as $\left[\mathrm{Vh}^{3}\right]$ in the $3 \mathrm{TS}\left(\mathrm{e} \cdot \mathrm{g} / \mathrm{ri}^{3{ }^{32}}+\mathrm{h}^{3} / \rightarrow\left[\mathrm{rif}^{3}\right]\right)$. $\left[\mathrm{CVh}^{3}\right]$ words are possible (e.g. $\left[j a \tilde{h} h^{3}\right]$ 'paper' - D:Table 4), so why $*\left[\beta \tilde{i} h^{3}\right]$ is not generated is unclear. Of course, as the $\left[\mathrm{h}^{3}\right]$ does not appear exactly when the root has a tone 3 , it is possible that a tonal OCP deletes the clitic entirely: e.g. $/ \beta \tilde{1}^{3}+\mathrm{h}^{3} / \rightarrow \mathrm{OCP}:\left[\beta \tilde{1}^{3}\right] \rightarrow$ lengthening: $\left[\beta \tilde{1}^{3}{ }^{3}\right]$. At this point, I am not sure how to integrate this observation with the rule system developed above. However, it is not obvious that it requires an exchange rule.

As for tone itself, $\mathrm{D}, \S 3.2 .3$ note that the $1 \mathrm{~s}$ causes different tonal alternations depending on its context: lowering in the potential aspect, and raising in the perfective. Clearly, a complete analysis of IT's phonology and morphology would require a thorough analysis of such alternations. Again, however, it is not obvious that any of the tonal processes require an exchange rule.

\section{The larger issue}

The discussion above recasts the issue about the existence of exchanges in terms of a specific theory: i.e. does SPE require exchange rules? While this recasting might strike some as a theoretician's sleight of hand, it emphasizes that descriptive/taxonomic goals and goals of cognitive scientists can be significantly different. Descriptive linguists are interested in how to describe a relationship between observed word forms in the common communicative behavior of a community. Cognitive scientists are interested in the phonological module's representations and computational capabilities. So, while IT might descriptively have an exchange, it does not follow that a phonological module is capable of an exchange transformation.

However, even theoreticians might object that the redefined question is too narrow. Surely there is a larger concept of 'exchange' that applies to all theories? Can we not define the issue in a pan-theoretical way rather than chunking it into separate questions for each theory? A reason that a pan-theoretical definition seems enticing is because SPE and its successors (including OT) all agree that there is a phonological module and that the module generates a particular type of output. Crucially, they also largely agree about what outputs look like - i.e. they share theories of output representation. So, defining an exchange in terms of relations between outputs seems possible.

However, as discussed in section 2, the theories themselves (at least the ones mentioned here) do not provide formal mechanisms for defining exchanges in terms of direct output-output relations, so such definitions must be provided via derivation. Theories like SPE and OT implement derivation very differently, and these differences are not superficial: they differ even in their conception of which derivational changes take place in an apparent exchange.

For example, the analysis above assumed two important input $\rightarrow$ output mappings: /anĩ $+\mathrm{h} / \rightarrow$ anĩ $\mathrm{h}]$ and /anĩ $+\mathrm{h} / \rightarrow$ ani: $]$. However, in some theories these mappings do not exist. For example, Lexical Phonology and Morphology (LPM - Kiparsky 1982) is very similar to SPE in terms of computational mechanisms. However, LPM employs multiple strata of computation. In fact, D (Table 8) proposes that processes involving enclitics apply at Stratum 2. By the end of the lexical stratum, it is possible that that final vowel lengthening has applied: i.e. /anĩ/ $\rightarrow$ [anĩ:]. Therefore, the 1S and 3TS are concatenated to forms that have a final long vowel, and never a short vowel. So, unlike SPE, in this theory there is never any in-stratum mapping /anĩ $+\mathrm{h} / \rightarrow$ anĩ $]$; Stratum 2's input is /anĩ: $+\mathrm{h} /$.

The nature of the exchange problem in this theory is therefore quite different from the problem for a single-level analysis in SPE. In the LPM analysis, the crucial mappings include /anĩ: $+\mathrm{h} / \rightarrow$ anĩ $\mathrm{h}]$ and there is never an underlying form /anĩ $+\mathrm{h} /$. In SPE, there is no mapping involving /anĩ: $+\mathrm{h} /$, but there is one involving /anĩ $+\mathrm{h} /$. In LPM, then, the analytical challenge is different from SPE: one must explain why the vowel shortens in /anĩ: $+\mathrm{h} / \rightarrow$ [anĩ $]$, but lengthens in $/$ anĩ $+\mathrm{h} / \rightarrow$ anĩ:] 
There is at least one LPM analysis that is substantially similar to the SPE one. It is sketched in (7); note the additional rule 'superheavy vowel shortening' $(\mathrm{V} \rightarrow[-\mathrm{long}] / \mathrm{C} \#)$.

\begin{tabular}{|c|c|c|}
\hline Stratum 2 input & /a.nĩ:+h/ & /a.nĩh+h/ \\
\hline Concatenation & anĩ:h & anĩhh \\
\hline Syllabification & a.nĩ:h & a.nĩfh \\
\hline Superheavy V shortening & a.nĩก & \\
\hline Epenthesis & & a.nĩ.hĩh \\
\hline onset fi deletion & & a.nĩ.ĩh \\
\hline VV merger & & a.nĩ:h \\
\hline Superheavy C deletion & & a.nĩ: \\
\hline Surface Form & a.nĩf & a.nĩ: \\
\hline
\end{tabular}

Concatenation of the input /a.nĩ: $+\mathrm{h} /$ creates a superheavy syllable: [a.nĩ:h]. To generate the attested output, the vowel must be shortened, producing [anĩf]. Without superheavy vowel shortening, the output would be *[anĩ:]. However, this derivation now requires two rules that resolve superheavy syllables: one that applies early in the derivation (i.e. before VV merger), and one that applies later in the derivation. The early rule resolves superheavy syllables by vowel shortening; the later rule resolves it by consonant deletion.

Of course, the analysis still generates the right outputs without use of an exchange rule. However, it is quite different in kind from the SPE analysis. Unlike the SPE analysis, extrinsic rule ordering is needed here (or the superheavy rules must be placed in different strata). The nature of the mappings are likewise quite different.

So, the question "does IT have a morphophonological exchange?" requires very different answers in SPE and LPM. Even though the theories have very similar computational mechanisms, the nature of the problem - i.e. their input $\rightarrow$ output derivations - is different.

The point is even more striking when we consider a theory with profoundly different computational mechanisms, like single level parallelist OT (Prince \& Smolensky 2004). In this framework, Alderete (2001) and de Lacy (2012) recast the exchange question as being about specific constraints, such as transderivational anti-faithfulness. So, asking whether IT requires an exchange mechanism within singlelevel parallelist OT translates into asking whether IT's system can only be generated by constraint systems that include transderivational faithfulness constraints.

The question of what exactly maps to what in IT is different again in OT. SPE analyses require accounting for /anĩ $+\mathrm{h} /$ but not /anĩ: $+\mathrm{h} /$; LPM worries about /anĩ $+\mathrm{h} /$ and not /anĩ $+\mathrm{h} /$. However, OT requires attention to both underlying forms due to Richness of the Base (see discussion in McCarthy 2002:76-81). If /anĩ:/ maps faithfully to [anĩ:], then /anĩ: $+h /$ must map to [anĩ $]$ ]. There are therefore three crucial mappings to worry about in OT: /anĩ $+\mathrm{h} / \rightarrow[\operatorname{ani\tilde {h}]}$, /anĩ: $+\mathrm{h} / \rightarrow$ [anĩ $]$, and $/$ anĩ $+h / h\left[\right.$ anĩ:] ${ }^{6}$ So, for some constraint component that lacks transderivational anti-faithfulness constraints, is there a ranking which will generate all of these mappings correctly?

Interestingly, single-level parallelist OT with Richness of the Base faces similar issues to the LPM rule system above: opacity mechanisms are necessary to generate the right outcome. Using Sympathy Theory (McCarthy 1999), /anĩh-h/ maps to [anĩ:] because the winner needs to preserve the loser [anĩfĩ

A ranking is sketched in (8); long vowels are shown as sequences of identical vowels to aid in tracking the vowel correspondences. The constraint MAX-C is crucial in singling out the opaque candidate [anĩhĩh] (the $*$ indicates that this constraint must be violated least by the opaque candidate). The constraint \& MAX$\mathrm{V}$ requires every vowel in the opaque candidate (marked with a 8 ) to appear in the winner. The crucial

\footnotetext{
${ }^{6}$ There are in fact a number of other underlying forms to worry about: e.g. /anĩ:h/, /anĩhh/, and so on. While all these forms must map to a grammatical output, I will restrict my attention to the more obvious mappings here.
} 
point here is that [a.nĩi] wins in (8) only because it can refer to [a.nĩ.fĩh], otherwise the transparent candidate *[a.nĩh] would win.

Sympathy analysis of /anĩh-h/ $\rightarrow$ [anĩ:]

\begin{tabular}{|c|c|c|c|c|c|}
\hline 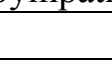 & /anĩh-h/ & $8 \mathrm{MAX}-\mathrm{V}$ & $* \mathrm{ONS} / \mathrm{h}$ & *SUPERHEAVY & *MAX-C \\
\hline a. 8 & a.nĩ.hĩh & & $* !$ & & \\
\hline b. & a.nกิ1ิh & & & $* !$ & $*$ \\
\hline c. & a.nĩh & $* !$ & & & $*$ \\
\hline d. & a.nกิ1 & & & & $* *$ \\
\hline
\end{tabular}

Tableau (9) shows how the ranking works for /anĩi-h/. Here, the winner [a.nĩh] is also the 'transparent' form - i.e. the form selected by the Sympathy mechanism.

(9) Sympathy analysis of /anĩ̄- $\mathrm{h} / \rightarrow$ anĩ $\mathrm{h}]$

\begin{tabular}{|c|c|c|c|c|c|}
\hline (n) & /anĩî-h// & $8 \mathrm{MAX}-\mathrm{V}$ & $*$ ONS/h & *SUPERHEAVY & $* \mathrm{MAX}-\mathrm{C}$ \\
\hline a. & a.nก̃ิโ & & & $* !$ & \\
\hline b. & a.nĩi & & & & $* !$ \\
\hline c. & a.nĩh & & & & \\
\hline
\end{tabular}

A fuller analysis would include constraints such as MAX-V (which necessarily ranks below * MAX-C, otherwise /anĩ1-h/ $\rightarrow^{*}[\operatorname{an} \tilde{1}]$ ), and DEP-V (which also must rank below ${ }^{2}$ MAX-C so that $/$ nano? $+h / \rightarrow$ [nano?of], rather than $*[$ nanof]). Even so, with this sketch of an analysis, transderivational anti-faithfulness constraints (which require outputs to have the opposite value of a feature when compared with their base) are not evidently necessary. So, the IT forms do not require anti-faithfulness constraints in single-level parallelist OT.

On the other hand, the IT mappings do seem to require an opacity mechanism in the OT analysis. So, suppose that we have an OT theory with no opacity mechanisms. Are exchange mechanisms necessary? Quite possibly - and this is in fact what D:§3.2.2 argue. Without exchange mechanisms, the challenge is to have /anĩh-h/ $\rightarrow$ [anĩ:]. An anti-faithfulness constraint like $\neg$ IDENT[spread glottis] "Incur a violation if there is not some base-derivative correspondent which differs in its value for [spread glottis]" might work (I assume here that the root $/ \mathrm{h} /$ can corresponds to an output vowel).

\begin{tabular}{|c|c|c|c|c|c|}
\hline & /anĩh-h/ (base: [anĩh $\left.{ }_{1}\right]$ ) & $\neg$ IDENT[sg] & $* \mathrm{ONS} / \mathrm{h}$ & *SUPERHEAVY & MAX-C \\
\hline a. & a.nĩ.ĥ1 & $* !$ & $* !$ & & \\
\hline b. & a.n1̃ ${ }_{1} h$ & & & $* !$ & $*$ \\
\hline c. & a.nĩh & $* !$ & & & $*$ \\
\hline d. & a.nก̃ĩ & & & & $* *$ \\
\hline
\end{tabular}

So, does IT require anti-faithfulness constraints in single-level parallelist OT? For a version of the theory without opacity mechanisms, apparently yes, otherwise no. Outside of a particular theory, the question of whether exchange mechanisms are necessary is undefined.

\section{Proving exchanges}

The discussion above illustrates how an apparent exchange is derived without exchange rules or antifaithfulness constraints. At this point, the reader might be wondering how to prove that exchange mechanisms actually exist.

The first step is to choose a theory and identify one's exchange mechanism. For SPE, it is exchange rules. 'Requiring an exchange rule' means that every rule system that could generate the forms in question includes an exchange rule, and there is no such rule system that lacks exchange rules. So, there are two tasks. One is to demonstrate that there is some rule system with an exchange rule that generates the observed 
forms. The other is to demonstrate that every such rule system includes an exchange rule - a much more complex task.

Let us apply the task to IT. Is there some rule system with an exchange rule that generates the observed forms? D assert that there is such a system: "The pattern in Itunyoso Triqui is ... a morphological exchange rule" $(\mathrm{D}, \S 1)$. However, no specific rule is formulated. In fact, there are significant challenges in providing an exchange rule for IT. If the $1 \mathrm{~S}$ is underlyingly /h/ (as assumed both here and in D - e.g. §3.2.2), the mapping $/$ anĩ $+h / \rightarrow$ anĩ $]$ does not involve any segmental transformation. Therefore, no exchange rule has applied.

An exchange rule analysis might be possible if one assumes that the $1 \mathrm{~S}$ is not underlying $/ \mathrm{h} /$, but that the morpheme itself is a rule (along the lines of Anderson 1992), and specifically an exchange rule. However, in the mapping /anĩ $+1 \mathrm{~S} / \rightarrow$ anĩ $\mathrm{h}]$, no feature value is changed - instead an entirely new segment is inserted. In other words, with an SPE analysis it is not clear that there is any rule system that includes an exchange rule that generates the IT alternations.

For LPM, the situation is different: with the mappings /anĩ: $+h / \rightarrow[\operatorname{anĩ} h]$ and /anĩ $h+h / \rightarrow[$ anĩ: $]$ there is an apparent exchange of vowel length and [ $\mathrm{h}]$. Inspired by a suggestion in an earlier draft of $\mathrm{D}$, one could invoke an exchange rule such as $[\alpha$ spread glottis $] \rightarrow[-\alpha$ spread glottis $] /]_{1 \mathrm{~s}}$. So, /anĩ̄ $+1 \mathrm{~S} / \rightarrow[$ anĩ $]$ because

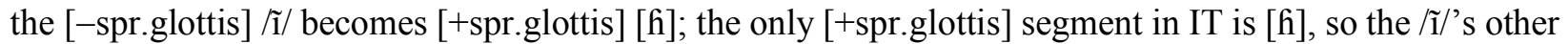
features must change accordingly. Similarly, /anĩh $+1 \mathrm{~S} /$ becomes [anĩi] because the [+spr.glottis] $/ \mathrm{h} /$ becomes [-spr.glottis] [î]. Presumably the /h/ does not become [?] because its [+continuant] feature must be preserved if possible. So, is an exchange rule analysis possible in LPM? I suspect so.

For OT, a similar point can be made. The derivational base of both /anĩ $+1 \mathrm{~S} /$ and /anĩ:+1s/ is [anĩ:]. So, $\neg$ IDENT[spr.glottis], which requires that the output have some segment which corresponds to a derivational base segment and differs in its value for [spr.glottis], can be invoked.

In other words, whether or not the first analytical hurdle can be made - i.e. to demonstrate that there is some computational system with an exchange mechanism - depends on the theory.

The other challenge is to demonstrate that, for one's theory, every computational system that generates the observed forms includes an exchange mechanism. D, $\$ 3.2 .1$ begin this task by providing a rule system that both lacks exchange rules and fails. In the D analysis, the $1 \mathrm{~S} / 3 \mathrm{TS}$ is underlyingly /-h/. However, concatenation of the $/ \mathrm{h} /$ is blocked by the OCP (which could be reconceived as OCP-driven C deletion): i.e. /anĩ $\mathrm{h}+\mathrm{h} / \rightarrow$ anĩ $\mathrm{h}]$. Then, there is root-final C-deletion so that [anĩ $\mathrm{h}] \rightarrow$ [anĩ], followed by final V lengthening to form [anĩ:]. In contrast, /anĩ $+h /$ does not undergo root-final $\mathrm{C}$ deletion, and so surfaces as [anĩf].

D point out some problems with this system, but the fatal problem actually has to do with deletion. I reformulate D's deletion rule as $\mathrm{C} \rightarrow \varnothing /]_{\text {root }}+\mathrm{C} \#$ here. ${ }^{7}$ However, the rule fails to apply in the derivation of /anĩ $h+h /:$ since the suffix $/ \mathrm{h} /$ is blocked/deleted by the OCP, the post-OCP output [anĩ $\mathrm{h}$ ] does not meet the structural description of the deletion rule, and so cannot then surface as [anĩ:].

D's analysis shows that there is at least one rule system that both lacks exchange rules and fails to generate the IT forms. However, how does one now demonstrate that all rule systems that lack exchange rules fail? To achieve this, the result from this rule system must be generalized to all rule systems: i.e. there must be some property of the rule system that all non-exchange-rule-containing rule systems have. Such demonstrations are difficult because they require a level of abstraction - of generalizing over rules and rule systems - that is often difficult to conceptualize (the same point can be made for arguments about exchangerelated constraints in OT, illustrated in de Lacy 2012). In this particular case, it might be possible to argue that all rule systems that include a deletion rule and lack an exchange rule fail. However, this result does not generalize to rule systems that include an epenthesis rule instead, as demonstrated in section 3. So, D's argument does not achieve the desired goal.

\footnotetext{
${ }^{7} \mathrm{D \# 22}$ 's deletion rule $\mathrm{C} \rightarrow \varnothing /$ ]root $_{\text {is }}$ inadequate because it incorrectly deletes all root-final consonants: e.g. /anĩh/ $\rightarrow *$ [anĩ] (and subsequently *[anĩ:]). The deletion rule cannot be saved by having it apply only in derived environments because /ni?jah-re?/ $\rightarrow$ [ni?jahre?] 'drink-2SG.FAM', not *[ni?jare?] (DiCanio 2008:154).
} 
D go further in the OT analysis in section $\$ 3.2 .2$, identifying a constraint set and ranking that fails to generate the attested forms, and generalizing the result: "The parallelist account cannot generate the correct output forms since it requires an exchange to take place within a single stage." D's insight here can be recast as an observation about faithfulness, assuming that there are no anti-faithfulness constraints. For the input /anĩh $+\mathrm{h} /$, the winner cannot be the attested [anĩ:] because [anĩ:] incurs a proper subset of [anĩh]'s IOfaithfulness violations ([anĩ:] violates IDENT-length and MAX twice; [anĩh] violates MAX once). Therefore, [anĩ:] could only win because some active markedness constraints favors it over [anĩf]. However, as D comment, "morphophonological exchanges are a challenge to classical, parallelist optimality-theoretic approaches because they necessarily involve a markedness paradox". In ranking terms, there is no possible ranking of markedness constraints that favors [anĩ:] over [anĩh] without also generating the wrong winners for /anĩ $+\mathrm{h} /$ or /nono? $+\mathrm{h} /$. For example, while NOCODA favors [anĩ $\mathrm{h}$ ] over [anĩ:], it will incorrectly favor [anĩ:] over [anĩ $]$ ] for the input /anĩ $+h /$. Of course, I only outline the argument here; even so, the thrust is clear: /anĩ $\mathrm{h}+\mathrm{h} / \rightarrow$ [anĩ:] requires a ranking of markedness constraints that is incompatible with /anĩ $+\mathrm{h} / \rightarrow[$ anĩ $]$.

So, D arguably do demonstrate that there is no ranking that both lacks exchange mechanisms (like antifaithfulness constraints) and generates the observed IT forms. However, it is crucial to be specific about the theory for which they have demonstrated this point: i.e. a single level parallelist theory that lacks opacity mechanisms. A two level theory, such as Stratal OT (Bermúdez-Otero 2018), could map /anĩ $+\mathrm{h} /$ to [anĩ fĩ $\mathrm{h}$ ] in Stratum 1 and then /anĩhĩ $/ \rightarrow$ anĩi] by capitalizing on preservation of vowels (i.e. MAX-V), adapting the LPM and OT analysis presented in section 4. Similarly, a single level parallelist theory that has opacity mechanisms (like Sympathy) can generate the IT forms, as shown in section 4.

Summing up, are the IT forms evidence that exchange mechanisms are necessary? As we have seen, the question is undefined outside the context of a specific theory. The answer, and even the nature of the problem, depends on the theory.

\section{Are all apparent exchanges possible?}

By refocusing the exchange mechanism issue on the derivation rather than outputs, it may seem that the door has been opened to observing any kind of apparent exchange in output forms. For example, could something similar to classic DhoLuo plural exchange - where [voice] values are apparently switched - ever be observed?

Unsurprisingly, the answer depends on the capability of the theory in question. To derive certain types of alternations, specific rule types and interactions are needed. For example, suppose there are input $\rightarrow$ output mappings $/ \mathrm{ap}+\mathrm{i}^{\mathrm{PL}} / \rightarrow$ abi] and $/ \mathrm{ab}+\mathrm{i}^{\mathrm{PL}} / \rightarrow$ [api] (PL marks the plural suffix). Is there any SPE rule system which lacks exchange rules yet can generate these mappings?

A general problem with such feature-value switching mappings is how to avoid neutralizing the distinction between the two derivations at any point in the derivation. For example, suppose there is a intervocalic voicing rule that maps $/ \mathrm{ap}+\mathrm{i}^{\mathrm{PL}} /$ to [abi]. A rule is now needed to devoice $/ \mathrm{b} /$ so that $/ \mathrm{ab}+\mathrm{i}^{\mathrm{PL}} / \rightarrow$ api] . But how does one now prevent such a devoicing rule from also applying to [abi]? Both /api/ and /abi/ would map to [api], and there would be no apparent exchange (i.e. $/ \mathrm{ap}+\mathrm{i}^{\mathrm{pL}} / \rightarrow$ voicing [abi] $\rightarrow$ devoicing [api] cf. $/ \mathrm{ab}+\mathrm{i}^{\mathrm{PL}} / \rightarrow$ devoicing [api]).

Any effective approach must make sure that /ap/ and /ab/ do not neutralize in the derivation. For example, a chain-shifting lenition process could map $/ \mathrm{ap}+\mathrm{i}^{\mathrm{PL}} / \rightarrow$ abi] and $/ \mathrm{ab}+\mathrm{i}^{\mathrm{PL}} / \rightarrow$ ami] (as in Irish eclipsis - Green 2006). A subsequent fortition process could then apply, where nasals become voiceless stops: $[\mathrm{am}] \rightarrow[\mathrm{ap}]$. The result is that underlying /ap/ becomes surface [ab], and underlying /ab/ becomes surface [ap], though via different derivational paths.

Does such a rule system mean DhoLuo-like exchanges will be observed? It depends on whether such rule systems are possible. The system sketched above involves a 'circular chain shift' of $/ \mathrm{p} / \rightarrow[\mathrm{b}] \rightarrow[\mathrm{m}] \rightarrow[\mathrm{p}]$. There is good reason to think that the phonological module is incapable of circular chain shifts, as discussed by Moreton (2004) and references cited therein. 
So, apparent exchanges whose derivation requires circular chain shifts are predicted to not be observed, as long as circular chain shifts are banned. Of course, it is quite possible that other apparent exchanges can be generated without circular chain shifts; IT provides an example. So, the issue of which apparent exchanges should be observed is one that will require a great deal more thought.

To add to the complexity of the problem for the analyst, exchange mechanisms do not necessarily generate visible $\mathrm{I} \rightarrow \mathrm{O}$ exchanges. For example, an exchange rule could condition a process, then neutralization could wipe out the change. An example is given in (11).

(11) Displacement effects of exchange rules

\begin{tabular}{l|l|l} 
Underlying form & $/ \mathrm{ap}+\mathrm{i}^{\mathrm{pL}} /$ & $/ \mathrm{ab}+\mathrm{i}^{\mathrm{p}} /$ \\
\hline$[\alpha$ voice $] \rightarrow[-\alpha$ voice] $/+\mathrm{PL}$ & a.bi & a.pi \\
\hline Vowel devoicing & & a.pi \\
\hline Stop devoicing & a.pi & \\
\hline Surface form & a.pi & a.pi
\end{tabular}

There is no visible feature exchange from the input to output here: /p/ and /b/ map to [p], and /i/ maps to [i] and [i] depending on the environment. Even so, an exchange rule has applied. In other words, in any serious quest for evidence for exchange rules, one must determine what their influence would be if obscured by later rules. In the derivation above, the effect is an 'unnatural' trigger: one would expect the voiceless stop to trigger devoicing, but the voiced stop does instead.

\section{The analyst's task}

The term 'exchange' means very different things in descriptive vs. theoretical contexts. For taxonomy/description, it might be a useful term to describe alternations where $[\alpha]$ in a stem corresponds to $[\beta]$ in its derived form, and $[\beta]$ in a stem corresponds to $[\alpha]$ in its equivalent derived form. For theoreticians, though, the term 'exchange' is a term of art: it is a particular type of derivation effected through specific computational mechanisms. For SPE, Anderson \& Browne (1973)'s mechanism is an exchange rule which effects derivations that include $[\ldots+\mathrm{F}]_{\mathrm{M}} \rightarrow[\ldots-\mathrm{F}]_{\mathrm{M}}$ and $[\ldots-\mathrm{F}]_{\mathrm{M}} \rightarrow[\ldots+\mathrm{F}]_{\mathrm{M}}(\mathrm{F}$ is a feature, $\mathrm{M}$ is a morpheme). For parallelist OT, exchange mechanisms are particular constraint types (such as transderivational anti-faithfulness and MORPHDISF) in the context of broader theories of the constraint component (de Lacy 2012).

From a taxonomic/descriptive point of view, IT does have an exchange because one observes bare root and 1s doublets such as [anĩ:] [anĩh] 'stop+1s' and [anĩh] $\rightarrow$ [anĩ:] 'get dirty+1s': i.e. [:] in the root corresponds to [G] in the $1 \mathrm{~S}$ and [G] in the root corresponds to [:] in the $1 \mathrm{~S}$.

What does IT tell us about specific phonological theories? For SPE, it appears possible to construct a rule system that generates the forms observed in IT without an exchange rule; it is certainly in principle possible for an SPE rule system to generate apparent exchanges without using exchange rules. So, one may conclude that - at this stage of knowledge about IT's morphology and phonology - there is no evidence for exchange rules here. For single-level parallelist Optimality Theory, it also seems likely that the IT alternations can be generated, perhaps relying on some opacity mechanism, but crucially without transderivational anti-faithfulness, or other exchange mechanisms discussed in de Lacy (2012).

Regardless, for the theoretician, it is clear that apparent exchanges pose a methodological challenge. Understanding such exchanges requires delving into the morphological and phonological system of the language, analyzing the broader morphological and phonological system, and then determining whether it generates the apparent exchanges without an exchange mechanism. It was only because D provided detail about the broader IT phonological system that it was possible to analyze the apparent exchanges in another way here. If $\mathrm{D}$ had only presented a few alternations without any larger context, any counter-proposal would have merely consisted of a shrug of uncertainty.

Even so, in my view, the information about IT that is currently available is not extensive enough to come to any conclusions with confidence. In the interim, the best that one can conclude is that there are 
some SPE rule systems (and OT constraint rankings) that do not include exchange mechanisms yet generate forms that look like exchanges. One such system might account for IT's apparent exchanges.

\section{References}

Alderete, John. 2001. Dominance effects as transderivational anti-faithfulness. Phonology 18. 201-253. https://doi.org/10.1017/S0952675701004067.

Anderson, Stephen R. 1992. A-morphous morphology. Cambridge, UK: Cambridge University Press.

Anderson, Stephen R. \& Wayles Browne. 1973. On keeping exchange rules in Czech. Papers in Linguistics 6. $445-482$.

Bermúdez-Otero, Ricardo. 2018. Stratal Phonology. In S. J. Hannahs \& Anna R. K. Bosch (eds.), The Routledge handbook of phonological theory. 100-134. Abingdon: Routledge.

Bye, Patrik. 2006. Eliminating exchange rules in Dholuo. Manuscript. University of Tromsø/CASTL. http://citeseerx.ist.psu.edu/viewdoc/download?doi=10.1.1.627.7938.

Chomsky, Noam and Morris Halle. 1968. The sound pattern of English. New York: Harper \& Row.

DiCanio, Christian T. 2008. The phonetics and phonology of San Martín Itunyoso Trique. Berkeley, CA: University of California dissertation.

DiCanio, Christian T. 2010. Itunyoso Trique. Journal of the International Phonetic Association 40.2. 227238. https://doi.org/10.1017/S0025100310000034.

DiCanio, Christian, Basileo Martínez Cruz, Benigno Cruz Martínez \& Wilberto Martínez Cruz. 2020. Glottal toggling in Itunyoso Triqui. Phonological Data and Analysis 2(4). 1-20. https://doi.org/10.3765/pda.v2art4.3.

Green, Anthony D. 2006. The independence of phonology and morphology: The Celtic mutations. Lingua 116(11). 1946-1985. https://doi.org/10.1016/j.lingua.2004.09.002.

Gregersen, Edgar A. 1974. Consonant polarity in Nilotic. In Erhard Voeltz (ed.), Third Annual Conference on African Linguistics. 105-109. Bloomington: Indiana University.

Hall, Nancy. 2011. Vowel epenthesis. In Marc van Oostendorp, Colin J. Ewen, Elizabeth Hume \& Keren Rice (eds.) The Blackwell companion to phonology, Volume 3. 1-21. Hoboken, NJ: Wiley-Blackwell. https://doi.org/10.1002/9781444335262.wbctp0067.

Kiparsky, Paul. 1982. Lexical morphology and phonology. In The Linguistic Society of Korea (ed.), Linguistics in the morning calm. 1-91. Seoul: Hanshin.

de Lacy, Paul. 2012. Morphophonological polarity. In Jochen Trommer (ed.), The morphology and phonology of exponence. 121-159. Oxford: Oxford University Press. https://doi.org/10.1093/acprof:oso/9780199573721.003.0005.

de Lacy, Paul and Patrik Bye. 2008. Metrical influences on lenition and fortition. In Joaquim de Carvalho, Tobias Scheer \& Philippe Ségéral (eds.), Lenition and fortition (Studies in Generative Grammar). 173206. Berlin: Mouton de Gruyter. https://doi.org/10.1515/9783110211443.

McCarthy, John J. 1999. Sympathy and phonological opacity. Phonology 16. 331-399. https://doi.org/10.1017/S0952675799003784.

McCarthy, John. 2002. A thematic guide to Optimality Theory. Cambridge University Press. https://doi.org/10.1017/CBO9780511613333.

McCarthy, John J. \& Alan S. Prince. 1995. Faithfulness and reduplicative identity. In Jill L. Beckman, Laura Walsh Dickey \& Suzanne Urbanczyk (eds.), Papers in Optimality Theory. 249-384. Amherst: GLSA Publications.

Moreton, Elliott. 2004. Non-computable functions in Optimality Theory. In John J. Mc-Carthy (ed.), Optimality Theory in phonology: A reader. 141-164. Hoboken, NJ: Wiley-Blackwell. https://doi.org/10.1002/9780470756171.

Prince, Alan S. \& Paul Smolensky. 2004. Optimality Theory: Constraint interaction in Generative Grammar. Hoboken, NJ: Wiley-Blackwell. https://doi.org/10.1002/9780470759400.

Shaw, Patricia A. 1982. Theoretical issues in Dakota phonology and morphology. London: Routledge. 
Trommer, Jochen. 2007. Voicing polarity in Luo. Manuscript. University of Leipzig. https://home.unileipzig.de/jtrommer/papers/antiauto6.pdf.

Wunderlich, Dieter. 2012. Polarity and constraints on paradigmatic distinctness. In Jochen Trommer (ed.), The morphology and phonology of exponence. 160-194. Oxford: Oxford University Press. https://doi.org/10.1093/acprof:oso/9780199573721.003.0006.

Paul de Lacy

Department of Linguistics

Rutgers University

18 Seminary Place

New Brunswick, NJ 08901, USA

delacy@rutgers.edu 